\title{
Spin-on doping of germanium-on-insulator wafers for monolithic light sources on silicon
}

\author{
Abdelrahman Z. Al-Attili ${ }^{1}$, Satoshi Kako ${ }^{2}$, Muhammad K. Husain ${ }^{1}$, F. Y. Gardes ${ }^{1}$, Hideo \\ Arimoto $^{1}$, Naoki Higashitarumizu ${ }^{3}$, Satoshi Iwamoto ${ }^{2}$, Yasuhiko Arakawa ${ }^{2}$, Yasuhiko \\ Ishikawa $^{3}$, and Shinichi Saito ${ }^{1 *}$ \\ ${ }^{I}$ Nano Research Group, Faculty of Physical Sciences and Engineering, The University of \\ Southampton, Southampton SO17 1BJ, U.K. \\ ${ }^{2}$ Institute of Industrial Science, The University of Tokyo, Meguro, Tokyo 153-8505, Japan \\ ${ }^{3}$ Department of Materials Engineering, Graduate School of Engineering, The University of \\ Tokyo, Bunkyo, Tokyo 113-8656, Japan \\ *E-mail: S.Saito@soton.ac.uk
}

High electron doping of germanium $(\mathrm{Ge})$ is considered to be an important process to convert Ge into an optical gain material and realize a monolithic light source integrated on a silicon chip. Spin-on doping is a method that offers the potential to achieve high doping concentrations without affecting crystalline qualities over other methods such as ion implantation and in-situ doping during material growth. However, a standard spin-on doping recipe satisfying these requirements is not yet available. In this paper we examine spin-on doping of Ge-on-insulator (GOI) wafers. Several issues were identified during the spin-on doping process and specifically the adhesion between $\mathrm{Ge}$ and the oxide, surface oxidation during activation, and the stress created in the layers due to annealing. In order to mitigate these problems, Ge disks were first patterned by dry etching followed by spin-on doping. Even by using this method to reduce the stress, local peeling of Ge could still be identified by optical microscope imaging. Nevertheless, most of the Ge disks remained after the removal of the glass. According to the Raman data, we could not identify broadening of the lineshape which shows a good crystalline quality, while the stress is slightly relaxed. We also determined the linear increase of the photoluminescence intensity by increasing the optical pumping power for the doped sample, which implies a direct population and recombination at the gamma valley. 


\section{Introduction}

Impurity doping of semiconductors is an indispensable process in the nanoelectronics industry to realize innovation of a wide variety of devices, including resonant tunneling diodes, ${ }^{1)}$ laser diodes (LD), ${ }^{2)}$ solar cells, ${ }^{3)}$ complimentary metal-oxidesemiconductor (CMOS), ${ }^{4,5)}$ and more. Multiple doping methods are available such as ion implantation, ${ }^{5,6)}$ in-situ doping, ${ }^{7}$ and spin-on-doping. ${ }^{8)}$ In particular, ion implantation is a paradigm in a standard CMOS process, ${ }^{6)}$ as it enables self-aligned doping for source and drain in MOS field-effect-transistors (FETs). ${ }^{5)}$ Recently, much attention has been drawn to achieve high level of donor doping in germanium $(\mathrm{Ge})$ for the fabrication of a $\mathrm{LD}$, monolithically integrated on a silicon ( $\mathrm{Si}$ ) substrate. ${ }^{9-12)} \mathrm{Ge}$ is an indirect band gap semiconductor, but by filling the indirect L valleys in the conduction band, we can observe direct recombination at the $\Gamma$ point. ${ }^{10)}$ A practical Ge LD is considered as the only missing component to realize a fully monolithically integrated Si photonics link, ${ }^{13)}$ as the other necessary photonic components, like high efficiency near-IR photo-detectors, ${ }^{14)}$ modulators, ${ }^{15)}$ and waveguides, ${ }^{16)}$ have been demonstrated and are well established. However, ion implantation may not be suitable for developing a Ge LD, due to the high implant dose, which will create an amorphous region at the surface of the material, while the bottom seed layer of epitaxially grown Ge contains a lot of dislocations. ${ }^{17,18)}$ These crystalline defects will work as non-radiative recombination centers and reduce the lightemission efficiency. ${ }^{13)}$ Therefore, it is necessary to develop a process for high donor doping in Ge while minimizing the damage in the crystal lattice, ${ }^{10)}$ and enable the fabrication of a high efficiency Ge LD. ${ }^{19)}$ In fact, lasing from Ge by optical pumping, ${ }^{20)}$ and electrical pumping, ${ }^{21)}$ were reported. However, so far, other research groups have not yet reproduced their results ${ }^{22)}$ in spite of the significant efforts in the last few years.

In order to convert slightly-strained Ge into an optical gain material, the active carrier concentration has to be above $5 \times 10^{19} \mathrm{~cm}^{-3}{ }^{23)}$ The solid solubility density of phosphorus $(\mathrm{P})$ in $\mathrm{Ge}$ is around $2 \times 10^{20} \mathrm{~cm}^{-3},{ }^{9)}$ so that, in principle, it is possible to use $\mathrm{P}$ to form the $n^{+}$Ge active layer of a LD. Furthermore, the tensile stress engineering of a Ge layer can reduce the required donor concentration to achieve the transparency condition. ${ }^{10,24)}$

One of the most straightforward techniques to achieve the high donor concentration is in-situ doping during the epitaxial growth of $\mathrm{Ge}$ on $\mathrm{Si}^{7,25)}$ However, there is a trade-off relationship between high crystalline quality and the doping concentration. ${ }^{7,25)}$ As a result, it is difficult to achieve doping concentration above $2 \times 10^{19} \mathrm{~cm}^{-3}$ without degradation of the crystal. ${ }^{7,25)}$ In order to overcome this problem, several approaches are proposed such as gas 
immersion laser doping (GILD) where a doping concentration of $5.6 \times 10^{19} \mathrm{~cm}^{-3}$ was achieved. ${ }^{12,26)}$ Another promising process is delta-doping of a Ge layer with a high concentration of $\mathrm{P}$ by low-temperature deposition on top of high-quality Ge. ${ }^{11)}$ The deltadoped layer works as a dopant source, and a doping concentration of $4 \times 10^{19} \mathrm{~cm}^{-3}$ was achieved. ${ }^{11)}$ The drawback of the delta-doping is the need to remove the delta-doped layer due to its poor crystalline quality. Such a layer has been shown to be successfully removed by employing chemical-mechanical polishing (CMP) process. ${ }^{11)}$

Another simple, yet promising method is spin-on doping (SOD) of Ge. ${ }^{27)}$ We expect minimum damage during SOD, since the process is based on diffusion of dopants. SOD has widely been used in near-infrared (IR) photo-detectors, ${ }^{28)}$ solar cells, ${ }^{8)}$ and MOSFETs ${ }^{29)}$ For a MOSFET, SOD of $\mathrm{P}$ with active carrier concentration of $7 \times 10^{19} \mathrm{~cm}^{-3}$ was achieved. ${ }^{29)}$ For this application, the sharp impurity profile is preferable for a high speed operation and a compact device size. On the other hand, for a light source, the uniform doping is preferable to make the whole Ge layer a positive gain medium, otherwise the optical loss from the lightly doped region compensates the gain. Nevertheless, Ge-on-Si, ${ }^{12)}$ or Ge-on-insulator $(\mathrm{GOI}),{ }^{12,30)}$ can be used to block the impurities diffusing out of $\mathrm{Ge}$, since the diffusion in $\mathrm{Ge}$ is much faster than in $\mathrm{Si}$ and oxide. More recently, successful doping of GOI has been reported and resonant photoluminescence (PL) from micro-disks at low temperatures has been observed. ${ }^{30)}$ This is a very promising achievement, and the disclosure of the details of their process conditions is highly awaited. Regardless of the apparent simplicity of the SOD process, many practical challenges have to be established for successful SOD of Ge, since the existing SOD sources were optimized for Si processing. ${ }^{28)}$ Therefore development of the SOD process is needed to identify challenges and establish a high electron doping process applicable for the fabrication of Ge LD, monolithically integrated on a Si chip.

In this work, we examine SOD processes by using two $\mathrm{P}$ dopant sources from different suppliers to dope commercially available GOI wafers. GOI wafers are useful to avoid defects generated at the interface of Ge and Si substrate during the growth. ${ }^{17)}$ This work defines and describes several practical problems and possible solutions to successfully apply SOD to GOI.

\section{Experimental methods}

We used commercially available GOI wafers with Ge thickness of $70 \mathrm{~nm}$ and buried-oxide (BOX) thickness of $145 \mathrm{~nm}$. After cleaning the samples by diluted hydrofluoric (HF) and hydrochloric $(\mathrm{HCl})$ acids, two groups of samples were prepared according to the 
type of P dopant sources used, as shown in Table I. Groups A and B contains P with identical concentration of $5 \times 10^{20} \mathrm{~cm}^{-3}$. This concentration exceeds the solid solubility of $\mathrm{P}$ in Ge, which should be enough to achieve a high active-carrier concentration with a uniform doping profile across the whole Ge thickness. ${ }^{8)}$ The main difference between the two dopant sources is the solvent, group A is a water-based solution of a polymer contatining $\mathrm{P}$, while group B is alcohol-based (ethanol) solution containing P. This affects the solution properties such as viscosity, which is crucial for spin-coating. According to data from suppliers, viscosity (at $25^{\circ} \mathrm{C}$ ) of the group A dopant source is $7 \mathrm{cP}$, while it is between $0.80-1.2 \mathrm{cP}$ for group B dopant.

A typical SOD process is shown in Fig. 1 below. After dispensing the spin-on dopant on the target wafer, it was then span-coated to form a uniform layer, soft-baked to evaporate solvents, and immediately annealed to diffuse and activate dopant impurities. Then, the SOD layer was removed by diluted HF etching. ${ }^{8,27-30)}$

\section{Process optimization}

Group A samples were coated with Emulsitone SOD film at the speed of $3000 \mathrm{rpm}$, and baked at $120^{\circ} \mathrm{C}$ for $15 \mathrm{~min}$ to evaporate the solvents. Temperature was increased to 120 ${ }^{\circ} \mathrm{C}$ with $10{ }^{\circ} \mathrm{C} / \mathrm{min}$ ramping rate, which was slow enough to prevent SOD film cracking. ${ }^{8}$ ) However, this film was highly non-uniform as confirmed by optical microscopy. The nonuniformity is clearly identified as line-shaped wrinkles corresponding to variations in film thickness. Surface profiler was used to map the thickness variation of this film along the center-line of the chip, and we found a variation of $\pm 60 \mathrm{~nm}$. This non-uniformity significantly affected the morphology of Ge after annealing due to the cracks. We expect that this cracking is due to stress from the SOD layer and the poor adhesion between Ge and $\mathrm{SiO}_{2}$. We could not deposit a $\mathrm{SiO}_{2}$ cap-layer by chemical vapor deposition (CVD), due to the nonuniformity. So, we span-coated spin-on-glass (SOG) solution with the thickness of $250 \mathrm{~nm}$ on top of the SOD layer. We examined two samples with different baking temperatures at 100 and $350{ }^{\circ} \mathrm{C}$, however, both samples were significantly damaged forming grains at the surface.

To solve this problem, we diluted the dopant source with isopropyl alcohol (IPA) solvent to achieve a better uniformity in the coating. Dilution with methanol was previously reported to control viscosity and thickness of spin-on dopant films. ${ }^{31)}$ Five solutions with SOD-to-IPA volume ratios of 1:1, 1:2, 1:5, and 1:10 were prepared, and the solutions were span-coated on the GOI samples at $3000 \mathrm{rpm}$. Resulting film thicknesses varied, accordingly, 
as shown in Fig. 2(a). The uniformity is improved with increased dilution, as confirmed by optical microscope images in Figs. 2(b) and 2(c). We annealed the five samples at $580{ }^{\circ} \mathrm{C}$ for $30 \mathrm{~min}$ in nitrogen $\left(\mathrm{N}_{2}\right)$. However, the surfaces of Ge were severely damaged after annealing except for the sample diluted to $1: 10$, which had a sheet resistance of $262 \Omega / \square$. Therefore, the 1:10 dilution was necessary to achieve the uniform coating and doping. We also examined the reduced activation time for 20 and $10 \mathrm{~min}$, and the resulting sheet resistances were 211 and $200 \Omega / \square$, respectively. In order to reduce the sheet resistance further, we repeated the SOD processes successively followed by the activation for $10 \mathrm{~min}$, after removing the SOD layer by HF at each time. The sheet resistance values after the second, third, and fourth doping repetitions, were 170,166 , and $168 \Omega / \square$, respectively. The resistivity was almost saturated after the second doping, which implies that the concentration of available dopant sources was not high enough after the dilution. The maximum doping concentration estimated by using the conductivity data ${ }^{32)}$ was around $1 \times 10^{19} \mathrm{~cm}^{-3}$. This estimation is done assuming that $\mathrm{P}$ distribution across the thickness of $\mathrm{Ge}$ is uniform, this is because the annealing time is long enough for $\mathrm{P}$ to diffuse across $70 \mathrm{~nm}$ of Ge. ${ }^{8)} \mathrm{In}$ spite of the successful doping confirmed by the sheet resistance measurements, we could not observe any PL signal from group A samples and could not identify the physical origins of the reduced PL signals even compared with the non-doped GOI.

We also used another solution, Filmtronics P507 (group B samples). We coated the solution at the ramp rates of 300,2000 , and $3000 \mathrm{rpm} / \mathrm{s}$, while keeping the same final speed at $3000 \mathrm{rpm}$. As shown in Fig. 3, the uniformities were affected by the ramp rate, and the best uniformity was achieved at the highest rate at $3000 \mathrm{rpm} / \mathrm{s}$ [Fig. 3(c)]. As a uniform coating was achieved by manipulating the coating-speed profile, dilution was not used for group B dopants. Then, we baked the sample at $120{ }^{\circ} \mathrm{C}$ for $15 \mathrm{~min}$, and annealed at $580{ }^{\circ} \mathrm{C}$ in $\mathrm{N}_{2}$ for $30 \mathrm{~min}$. Regardless of the apparent uniformity of the SOD layer before the annealing [Fig. 3(c)], the Ge layer was completely damaged after annealing and we could not measure the sheet resistance. The damage presumably originated from the stress of the SOD layer and the poor adhesion between Ge and BOX. Therefore, we could not dope unpatterned GOI using P507.

In order to mitigate these problems, we dry etched Ge first to make patterns including disks before the SOD process, as shown in Figs. 4(a) and 4(c). The diameter of the disks was $10 \mu \mathrm{m}$. It was expected that the stress could be released in isolated patterned structures, whereas the local relaxation would be difficult in the case of the film without making cracks. Then, we span-coated the SOD layer with the ramp rate of $3000 \mathrm{rpm} / \mathrm{s}$. We 
prepared two samples, annealed at $580{ }^{\circ} \mathrm{C}$ in $\mathrm{N}_{2}$ for 10 and $30 \mathrm{~min}$. In the sample annealed for $10 \mathrm{~min}$, we found gaseous bubbles dispersed in the SOD layer (not shown), whereas we could not observe the bubbles in the sample annealed for $30 \mathrm{~min}$. As shown in Fig. 4(b), we recognized that Ge was dissolved in the SOD layer, which smeared the edges of the original Ge structures. We consider that the surface of GOI was oxidized by the SOD layer during the activation, and the bubbles found would be $\mathrm{GeO}$ sublimated during the annealing. In fact, we also confirmed that the surface of Si was oxidized after SOD by measuring the thickness of Si-on-insulator (SOI) layer using spectroscopic ellipsometry. After removing the SOD layer, the GOI layer was partially peeled off, but the most of the disks survived [Fig. 4(d)]. Measuring the sheet resistance was not possible as we patterned the Ge layer before SOD, in order to reduce the stress on Ge during annealing.

\section{Results and discussion}

In this section, we discuss the properties of the doped Ge disk [sample B, Fig. 4(d)] compared with the Ge disk without doping. In order to examine the impact of SOD on crystalline qualities of $\mathrm{Ge}$, we measured Raman spectrum using a laser at the wavelength of $532 \mathrm{~nm}$ with the spot size of about $2 \mu \mathrm{m}$. The Ge disk was excited with pump power of 275 $\mu \mathrm{W}$ for $5 \mathrm{~s}$ using a 3000 lines/mm grating, and the signal was averaged over 10 repetitions to enhance signal-to-noise ratio. Figure 5 shows the Raman spectra measured at the center of the Ge disks before and after SOD. Experimental data were fitted by using Lorentzian forms. The peak position of the undoped Ge disk was found at the wavelength of $300.1 \mathrm{~cm}^{-}$ 1 , which shows the accumulation of the tensile strain compared with the wavelength of 301 $\mathrm{cm}^{-1}$ for the unstrained bulk single crystalline Ge. ${ }^{29,33)}$ This tensile strain would be presumably coming from the growth of Ge on a Si substrate ${ }^{18)}$ before transferring to the GOI substrate. The peak position of the doped disk was found to be at a wavelength of 300.33 $\mathrm{cm}^{-1}$, which is slightly blue-shifted after the thermal treatment at $580{ }^{\circ} \mathrm{C}$ for activation. The relaxation of the tensile strain could be induced, because of its patterned structure before annealing. On the other hand, the change of the peak width was negligible, implying a good crystalline quality of Ge with negligible local stress variations after SOD, in spite of visible voids in the disk [Fig. 4(d)]. We also found 5\% decrease of the intensity, which would be attributed to the decrease of the area due to the voids.

We measured PL at room-temperature by using a laser with a wavelength of 740 $\mathrm{nm}$ and a spot size of about $10 \mu \mathrm{m}$. PL spectra from intrinsic and doped Ge disks are shown in Figs. 6(a) and 6(b), respectively. The intensity of doped sample was higher than that of 
intrinsic one, which shows the increase in the recombination rate by the increase of the electron occupation at the L valleys ${ }^{9,12,23,34,35)}$ after the SOD process. The peak position for the doped sample was red-shifted by $5.24 \mathrm{~nm}$, and this is consistent with the direct band gap narrowing by doping. ${ }^{35)}$ The red-shift of the spectra cannot be explained by the relaxation of the stress, since a blue-shift is expected due to the relaxation of the tensile stress observed by Raman (Fig. 5). At the excitation power of $10 \mathrm{~mW}$, full-width at half-maximum (FWHM) linewidths of intrinsic and doped samples were $117 \mathrm{~nm}$ and $129 \mathrm{~nm}$, respectively. The larger FWHM of the doped sample implies a local variations of the carrier concentration. Comparing with reported PL intensity enhancement and redshift in peak position, ${ }^{12,23)}$ we estimate that the active doping concentration would be $(1-2) \times 10^{19} \mathrm{~cm}^{-3}$. This value is much less than solid solubility density of $\mathrm{P}$ in Ge, so that it will be possible to increase it further by optimizing the process conditions and structures, especially by improving the adhesion between $\mathrm{Ge}$ and $\mathrm{BOX}$. For instance, the use of thin $\mathrm{Al}_{2} \mathrm{O}_{3}$ layer between $\mathrm{Ge}$ and $\mathrm{BOX}^{17)}$ may enhance the adhesion. We did not observe the whispering-gallery-modes of the disks, ${ }^{30)}$ since our BOX layer was too thin to confine the optical modes within the GOI layer.

Figure 7 shows the peak PL intensities dependence on the excitation power, $P$, for intrinsic and doped Ge disks. The intensities for the doped sample depends linearly on $P$. This means the successful filling of the in-direct $L$ valleys in the conduction band by doping and the linear increase of the population of electron-hole pairs at the direct $\Gamma$ point by pumping. ${ }^{9,17,27)}$ On the other hand, the intensities for the intrinsic sample were proportional to $P^{2}$.

This remarkable difference can be explained by using the rate equation for the hole density, $p$, at the steady state ${ }^{36)}$

$$
\frac{d p}{d t}=P-A p-B n p=0
$$

where $A$ and $B$ are coefficients for non-radiative and radiative recombination rates, respectively, and $n$ is the electron density. The photon density of PL, $\gamma$, is obtained by

$$
\gamma=\beta B n p
$$

where $\beta$ is the extraction efficiency to the detector. For the doped sample, the $\mathrm{L}$ valleys were already occupied by the doped electrons with the constant density of $n_{0}$, which would be much larger than the excited hole density. Therefore, we can approximate that the electron density was almost constant $n \sim n_{0}$, independent of the pump power. Then, we obtain the linear dependence, 


$$
\gamma=\beta \frac{B n_{0}}{A+B n_{0}} P \propto P
$$

Note that this Eq. (3) is valid independent on the magnitudes of $A$ and $B$, so that the linear dependence cannot prove that the radiative recombination dominates over non-radiative processes. Essentially, the linear dependence of the doped sample was coming from the linear increase of the hole density by pumping, while the electron density was almost constant. On the other hand, for the intrinsic sample, the electron density agreed with the hole density, $n=p$, due to the charge neutrality, and assuming that the non-radiative recombination dominates over the radiative recombination, we obtain

$$
\gamma=\beta \frac{B}{A^{2}} P^{2} \propto P^{2}
$$

Therefore, the quadratic dependence is due to the fact that the recombination is the product of $n$ and $p$ [Eq. (2)], while these densities linearly depend on $P$, due to the short lifetime coming from the non-radiative recombinations. ${ }^{19)}$

This marked difference of the $P$ dependence makes it difficult to define the enhancement factor of the PL by doping, since the factor depends on P. Physically, the intensities of doped and intrinsic samples would eventually be comparable, if we excite the samples with enough power to allow the complete occupation of the $\mathrm{L}$ valleys even in the intrinsic sample. Our excitation power was not high enough to confirm this, since further increase of $P$ resulted in burning of the sample surface and sublimation of Ge was observed. In order to make Ge a positive gain material, higher doping concentration is necessary to achieve the population inversion with threshold accessible by optical pumping.

\section{Conclusions}

In conclusion, we have examined spin-on doping processes for Ge light emissions using two commercially available phosphorous dopant sources and Ge-on-insulator substrates. We have shown that spin-on doping of Ge is not straightforward, and there are many practical process issues still to be solved. In this work, most of the problems arose from the weak adhesion between Ge and the buried oxide layer. Great care must also be taken of the surface oxidation occurring during the annealing process as well as considerable stress generated by spin-on dopant layer. We considered several approaches to mitigate the issue of thermal stress. In particular, uniform coating was very important to avoid the formation of cracks 
during annealing. It was possible to dope Ge disks after patterning, while maintaining a reasonably good crystalline quality. We found a remarkable change in the excitation power dependence of the photoluminescence before and after doping, from quadratic to linear. We believe that spin-on doping processes are very promising to deliver higher activation levels suitable for realizing Ge-based active photonic devices on Si chips.

\section{Acknowledgments}

Parts of the studies discussed here were supported by the Japan Society for the Promotion of Science (JSPS) through its "Funding Program for World-Leading Innovation R\&D on Science and Technology (FIRST Program)," the Project for Developing Innovation Systems, and KAKENHI 216860312, the Ministry of Education, Culture, Sports, Science, and Technology (MEXT), Japan. This work is also supported by Marie-Curie Carrier Integration Grant (CIG, PCIG13-GA-2013-618116, FP7, EU), Standard Grant (EP/M009416/1, EPSRC, UK), and University of Southampton, Zepler Institute, Research Collaboration Stimulus Fund. The raw data from this paper can be obtained from the University of Southampton ePrints research repository, DOI: 10.5258/SOTON/377534.

\section{References}

1) L. Esaki, Phys. Rev. 109, 603 (1958).

2) R. N. Hall, G. E. Fenner, J. D. Kingsley, T. J. Soltys, and R. O. Carlson, Phys. Rev. Lett. 9, 366 (1962).

3) D. M. Chapin, C. S. Fuller, and G. L. Pearson, J. Appl. Phys. 25, 676 (1954).

4) F. M. Wanlass, and C. T. Sah, 6th Int. Solid-State Circuits Conf. (ISSCC) Tech. Dig., 1963, p. 32.

5) R. H. Dennard, F. H. Gaensslen, H.-N. Yu, V. L. Rideout, E. Bassous, and A. R. LeBlanc, IEEE J. Solid-State Circuits 9, 256 (1974).

6) Ch. Buchal, S. P. Withrow, C. W. White, and D. B. Poker, Annu. Rev. Mater. Sci. 24, 125 (1994).

7) H.-Y. Yu, E. Battal, A. K. Okyay, J. Shim, J.-H. Park, J. W. Baek, and K. C. Saraswat, Curr. Appl. Phys. 13, 1060 (2013).

8) N. E. Posthuma, J. Van der Heide, G. Flamand, and J. Poortmans, IEEE Trans. Electron Devices 54, 1210 (2007).

9) J. Liu, L. C. Kimerling, and J. Michel, Semicond. Sci. Technol. 27, 094006 (2012). 
10) J. Liu, X. Sun, D. Pan, X. Wang, L. C. Kimerling, T. L. Koch, and J. Michel, Opt. Express 15, 637 (2007).

11) R. E. Camacho-Aguilera, Y. Cai, J. T. Bessette, L. C. Kimerling, and J. Michel, Opt. Mater. Express 2, 1462 (2012)

12) M. El Kurdi, T. Kociniewski, T.-P. Ngo, J. Boulmer, D. Debarre, P. Boucaud, J. F. Damlencourt, O. Kermarrec, and D. Bensahel, Appl. Phys. Lett. 94, 191107 (2009).

13) S. Saito, K. Oda, T. Takahama, K. Tani, and T. Mine, Appl. Phys. Lett. 99, 241105 (2011).

14) J. Michel, J. Liu, and L. C. Kimerling, Nat. Photonics 4, 527 (2010).

15) J. E. Roth, O. Fidaner, R. K. Schaevitz, Y.-H. Kuo, T. I. Kamins, J. S. Harris, and D. A. B. Miller, Opt. Express 15, 5851 (2007).

16) G. Z. Mashanovich, M. M. Milosevic, M. Nedeljkovic, N. Owens, B. Xiong, E. J. Teo, and Y. Hu, Opt. Express 19, 7112 (2011).

17) D. Nam, J.-H. Kang, M. L. Brongersma, and K. C. Saraswat, Opt. Lett. 39, 6205 (2014).

18) Y. Ishikawa, K. Wada, J. Liu, D. D. Cannon, H.-C. Luan, J. Michel, and L. C. Kimerling, J. Appl. Phys. 98, 013501 (2005).

19) S. Saito, F. Y. Gardes, A. Z. Al-Attili, K. Tani, K. Oda, Y. Suwa, T. Ido, Y. Ishikawa, S. Kako, S. Iwamoto, and Y. Arakawa, Front. Mater. 1, 15 (2014).

20) J. Liu, X. Sun, R. Camacho-Aguilera, L. C. Kimerling, and J. Michel, Opt. Lett. 35, 679 (2010).

21) R. E. Camacho-Aguilera, Y. Cai, N. Patel, J. T. Bessette, M. Romagnoli, L. C. Kimerling, and J. Michel, Opt. Express 20, 11316 (2012).

22) L. Carroll, P. Friedli, S. Neuenschwander, and H. Sigg, Phys. Rev. Lett. 109, 057402 (2012).

23) J. T. Bessette, R. Camacho-Aguilera, Y. Cai, L. C. Kimerling, and J. Michel, 8th IEEE Int. Conf. Group IV Photonics (GFP), 2011, p. 130.

24) M. El Kurdi, G. Fishman, S. Sauvage, and P. Boucaud, J. Appl. Phys. 107, 013710 (2010).

25) J. M. Hartmann, J. P. Barnes, M. Veillerot, J. M. Fédéli, Q. B. A. L. Guillaume, and V. Calvo, J. Cryst. Growth 347, 37 (2012).

26) D. Cammilleri, F. Fossard, D. Débarre, C. Tran Manh, C. Dubois, E. Bustarret, C. Marcenat, P. Achatz, D. Bouchier, and J. Boulmer, Thin Solid Films 517, 75 (2008).

27) J. Liu, R. Camacho-Aguilera, J. T. Bessette, X. Sun, X. Wang, Y. Cai, L. C. Kimerling, and J. Michel, Thin Solid Films 520, 3354 (2012).

28) V. Sorianello, A. De Iacovo, L. Colace, A. Fabbri, L. Tortora, and G. Assanto, Phys. 
Status Solidi 11, 57 (2014).

29) M. Jamil, J. Mantey, E. Onyegam, G. Carpenter, E. Tutuc, and S. Banerjee, IEEE Electron Device Lett. 32, 1203 (2011).

30) X. Xu, K. Nishida, K. Sawano, T. Maruizumi, and Y. Shiraki, Tech. Dig. Conf. Lasers and Electro-Optics (CLEO), 2014, SM4H.3.

31) D. Mathiot, A. Lachiq, A. Slaoui, S. Noel, J. C. Muller, and C. Dubois, Mater. Sci. Semicond. Process. 1, 231 (1998).

32) D. B. Cuttriss, Bell Syst. Tech. J. 40, 509 (1961).

33) C.-Y. Peng, C.-F. Huang, Y.-C. Fu, Y.-H. Yang, C.-Y. Lai, S.-T. Chang, C. W. Liu, J. Appl. Phys. 105, 083537 (2009).

34) X. Sun, J. Liu, L. C. Kimerling, and J. Michel, Appl. Phys. Lett. 95, 011911 (2009).

35) R. Camacho-Aguilera, Z. Han, Y. Cai, L. C. Kimerling, and J. Michel, Appl. Phys. Lett. 102, 152106 (2013).

36) M. A. Parker, Physics of Optoelectronics (CRC Press, Boca Raton, FL, 2005). 


\section{Figure Captions}

Fig. 1. SOD process steps: (a) dispense spin-on-dopant source, (b) spin-coating using an optimized speed-profile, (c) soft-baking to solidify dopant layer by evaporating solvents, (d) diffusion and activation by annealing, and followed by (e) removal of depleted SOD film by diluted HF etching.

Fig. 2. Dilution of spin-on dopants using IPA: (a) thickness variation according to dilution percentage, (b) optical microscopy of Emulsitone $\left(5 \times 10^{20} \mathrm{~cm}^{-3}\right)$ spin-on-dopant coated at $3000 \mathrm{rpm}$ before dilution, and (c) 1:10 SOD:IPA coating using same conditions.

Fig. 3. Optical microscopy images of Filmtronics P507 spin-on-dopant film coated using three different speed-profiles, with acceleration values chosen as: (a) 300, (b) 2000, and (c) $3000 \mathrm{rpm} / \mathrm{s}$.

Fig. 4. Optical microscopy images of group B samples before and after SOD annealed at $580{ }^{\circ} \mathrm{C}$ : array of Ge lines (a) before and (b) after annealing for $30 \mathrm{~min}$. Image (b) was taken before removing the SOD layer. $10 \mu \mathrm{m}$ micro-disks (c) before and (d) after SOD annealed for 30 min. Image (d) was taken after removing the SOD layer.

Fig. 5. Raman spectra of intrinsic (blue) and doped (red) Ge disk, annealed at $580{ }^{\circ} \mathrm{C}$ for 30 min in nitrogen. The relaxation of tensile stress after annealing was found.

Fig. 6. Photoluminescence spectra of (a) intrinsic Ge disk and (b) doped Ge disk annealed at $580{ }^{\circ} \mathrm{C}$ for $30 \mathrm{~min}$ in nitrogen. After doping, broadening of spectra and red-shift were found, implying carrier non-uniformities and band gap narrowing.

Fig. 7. Peak photoluminescence intensities dependence on excitation power for intrinsic Ge disk (black) and doped Ge disk (red). The lines are quadratic and linear fits to experimental data. 
Table I. Groups of samples according to the type of dopants.

\begin{tabular}{ccccc}
\hline Group & Dopant supplier & Product & $\begin{array}{c}\text { P concentration } \\
\left(\mathrm{cm}^{-3}\right)\end{array}$ & $\begin{array}{c}\text { Thickness at } \\
3000 \mathrm{rpm}(\mathrm{nm})\end{array}$ \\
\hline A & Emulsitone & Phosphorosilicafilm & $5 \times 10^{20}$ & 380 \\
B & Filmtronics & P507 & $5 \times 10^{20}$ & 230 \\
\hline
\end{tabular}




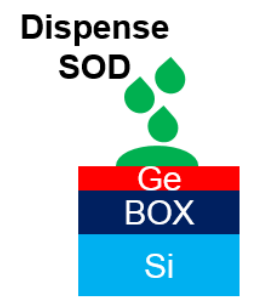

(a)

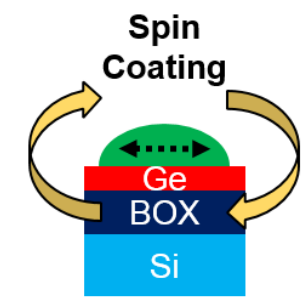

(b)

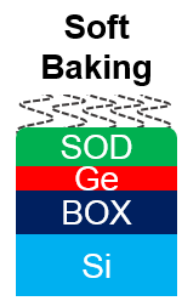

(c)

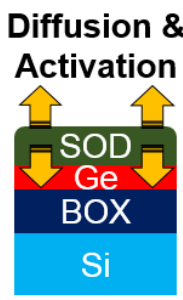

(d)
SOD

Removal

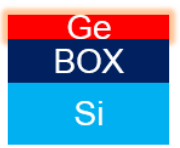

(e)

Fig. 1. 


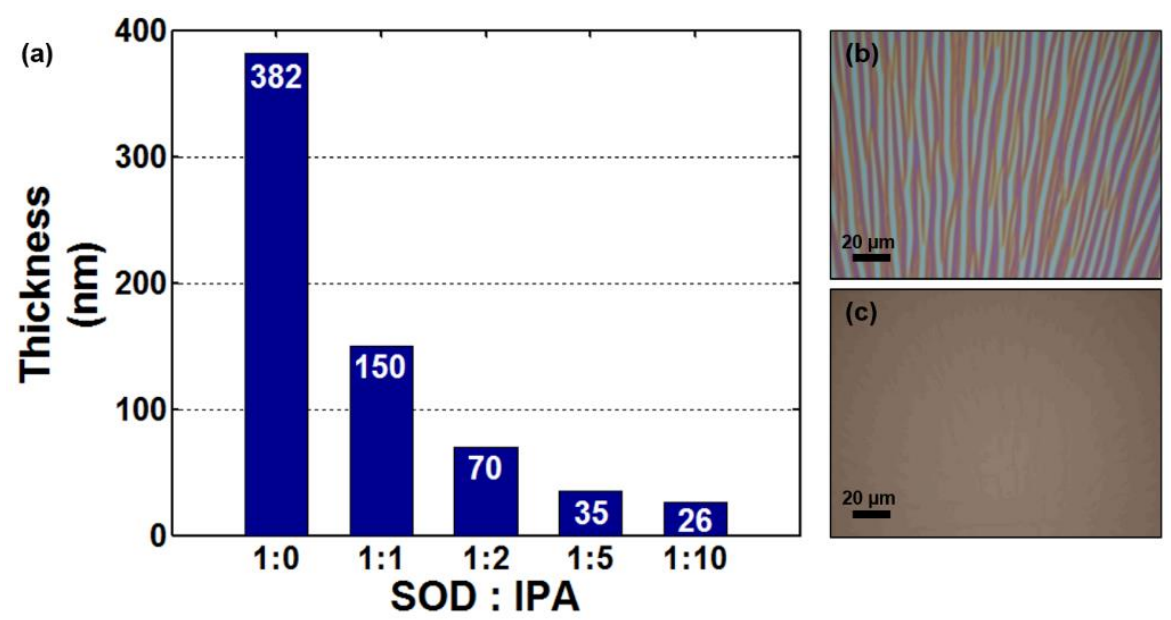

Fig. 2. 


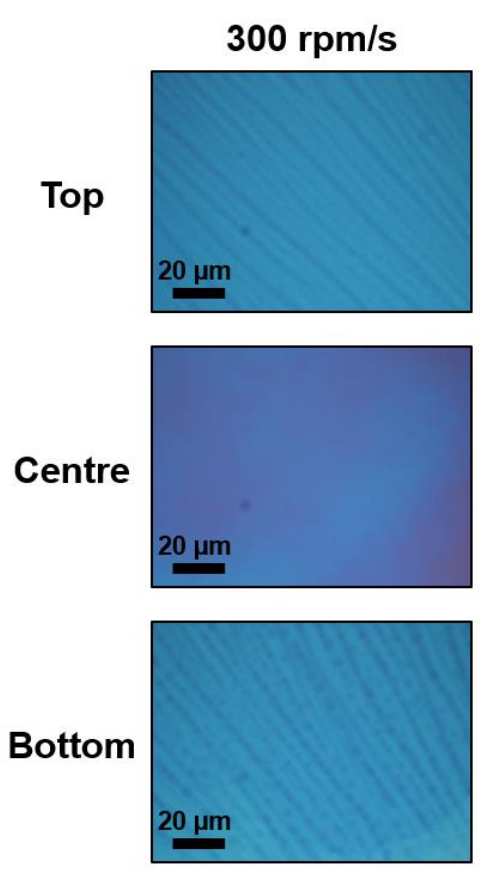

(a)
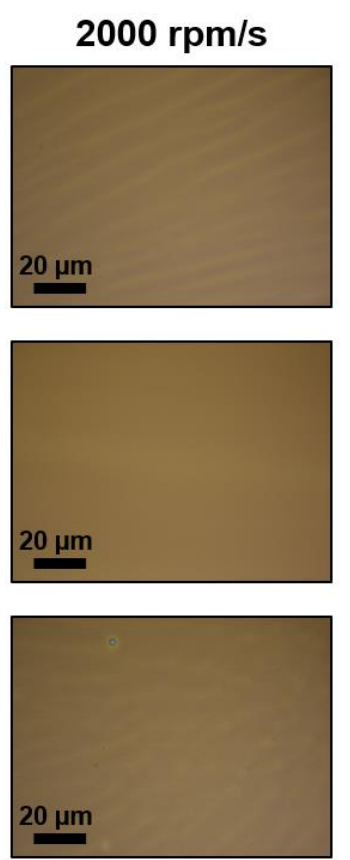

(b)
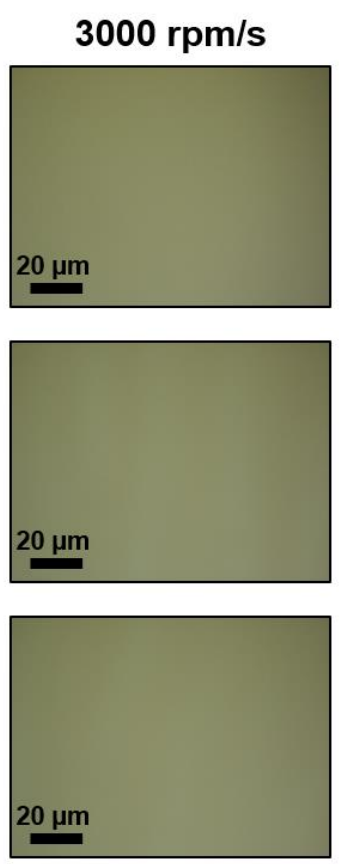

(c)

Fig. 3. 

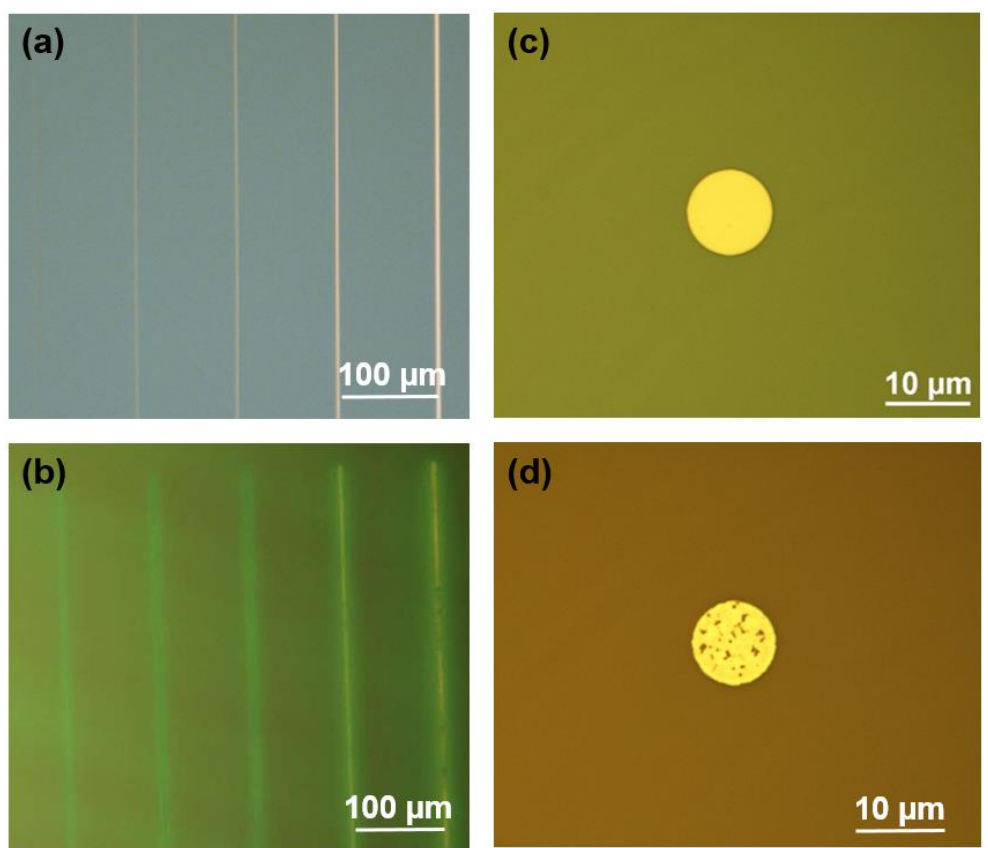

Fig. 4. 


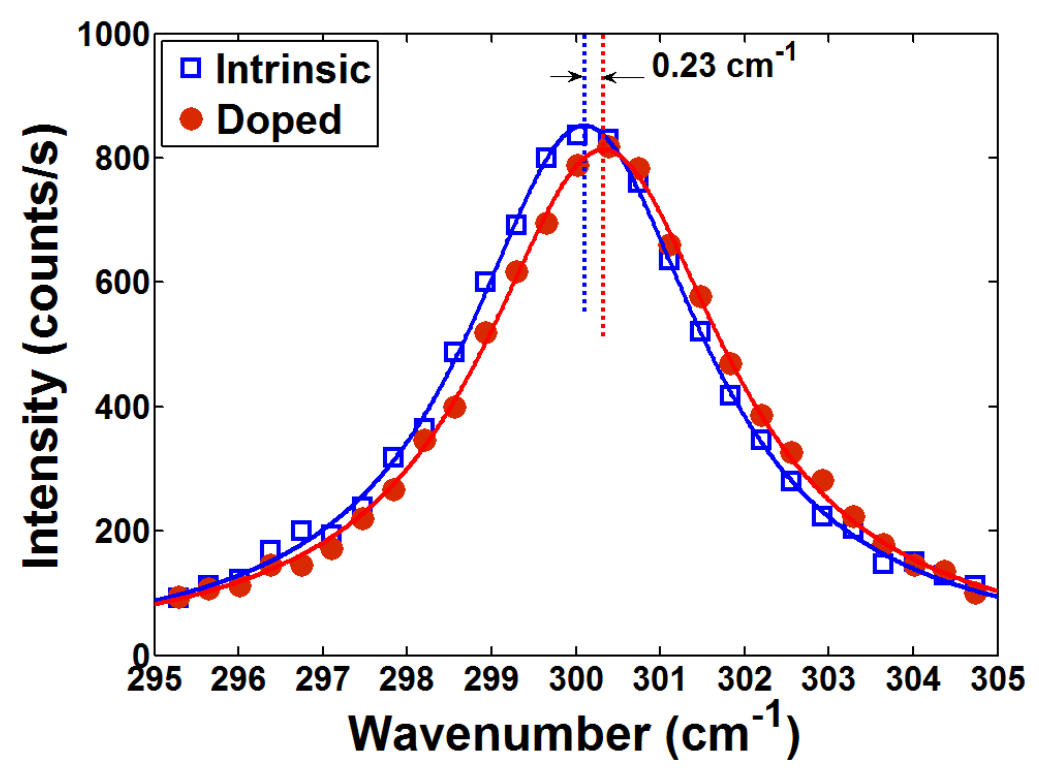

Fig. 5. 

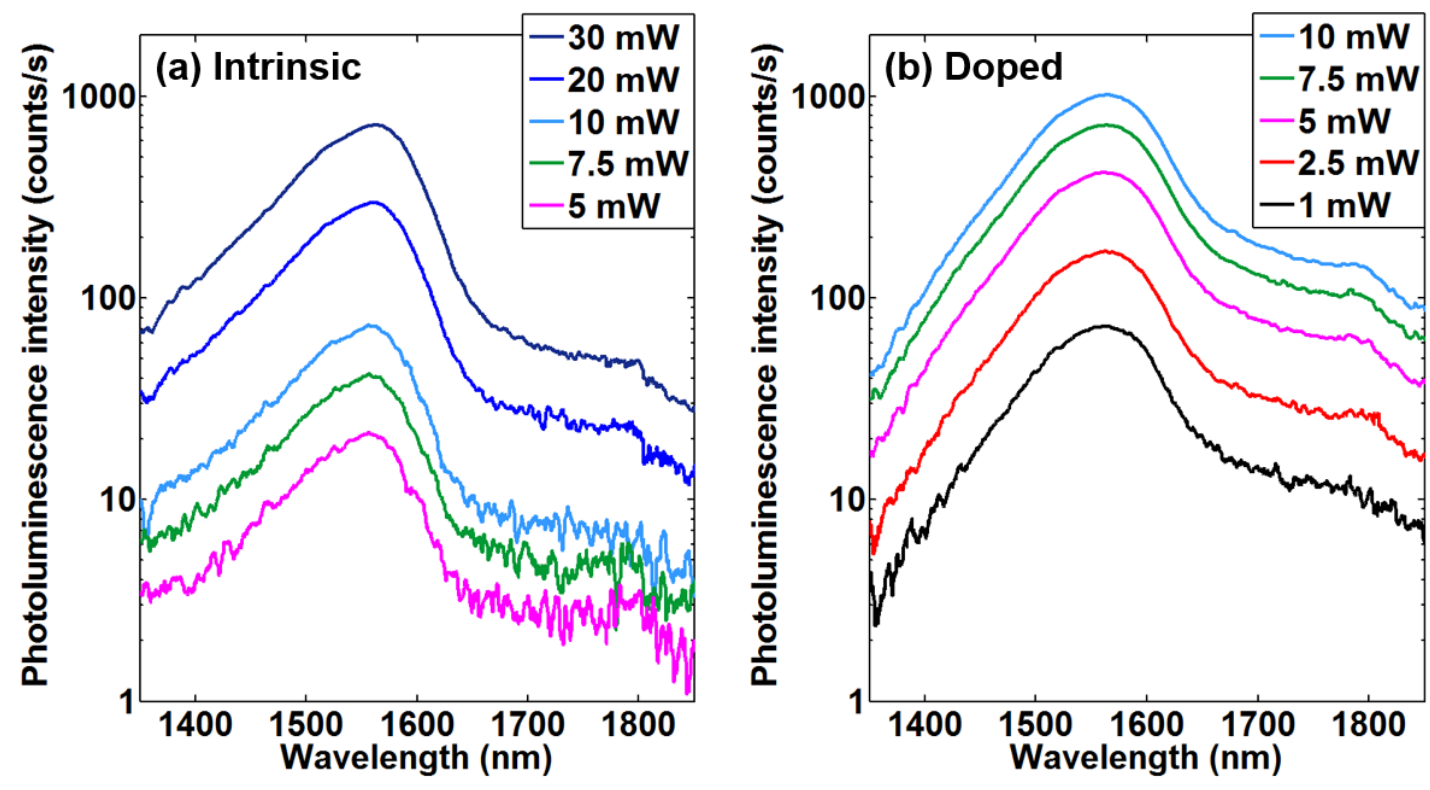

Fig. 6. 


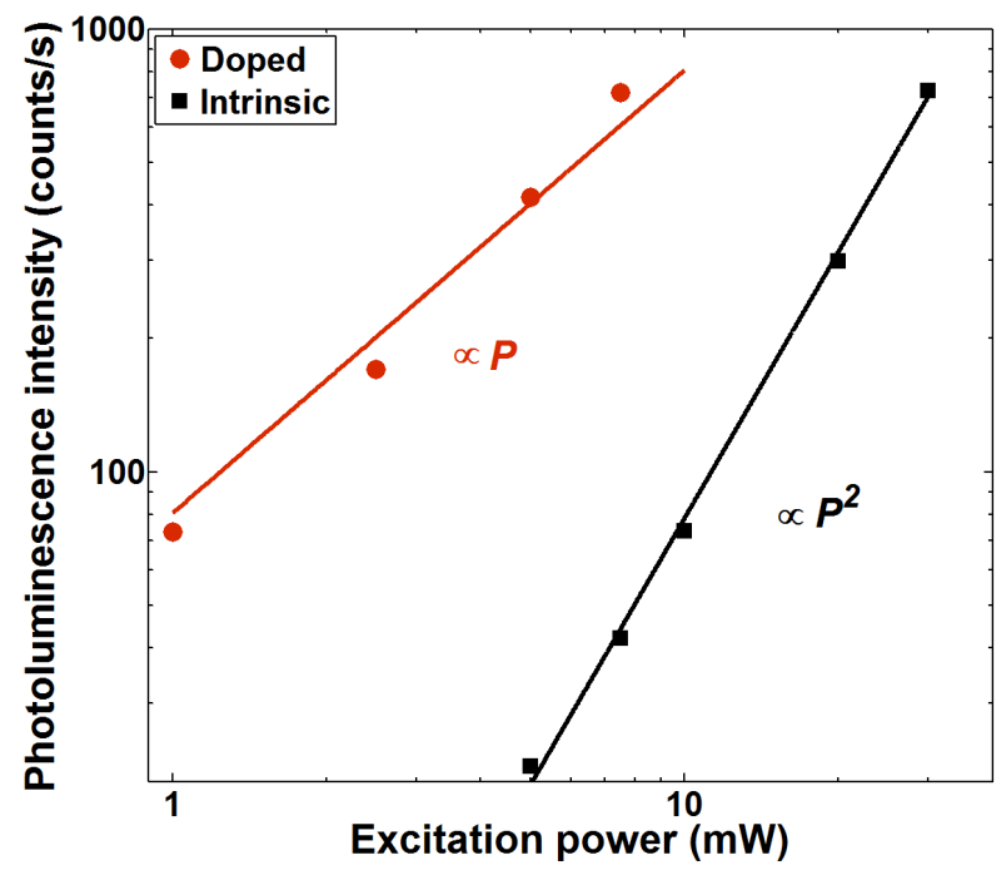

Fig. 7. 